Jörg Potthast

\title{
Politische Soziologie technischer Prüfungen
}

\author{
Das Beispiel Straßenverkehrssicherheit
}

\section{Sicherheit und Symbolpolitik}

Auch wenn sie von den Herstellern aus verständlichen Gründen immer wieder zurückdatiert wurde, beginnt die Geschichte der automobilen Sicherheit in den USA erst im Jahre 1966. ${ }^{1}$ Damals kam es nach der sehr erfolgreichen, von Ralph Nader, dem Verbraucheranwalt, initiierten Kampagne »Unsafe at any speed « (so auch der Titel seines Buchs) erstmals zu strengeren gesetzlichen Auflagen für passive Sicherheit. Einige europäische Autokonzerne haben seitdem die US-amerikanischen Sicherheitsvorschriften auch für Absatzmärkte übernommen, die weniger strengen Zulassungsvorschriften unterlagen.

Über einen Werbeclip der Firma Renault erhielt 40 Jahre später ein verbraucherpolitisches Instrument ein ungewohnt publikumswirksames Format: Unschwer als Nationalstereotype erkennbare Artefakte (Sushi, Knäckebrot, Weißwurst, Baguette) werden in einer Versuchsanlage einem Crashtest unterzogen. Das Resultat ist evident und in der Zeitlupe spektakulär: Mit Ausnahme des Baguette, das den Aufprall leicht geknautscht übersteht, verlieren alle Testobjekte ihre Form. Der Konzern wirbt hier nicht nur mit guten Testergebnissen, sondern re-inszeniert den ganzen Test: In einem Feld, in dem Ingenieurskulturen so disparat sind und so weit auseinanderliegen wie kulinarische Traditionen, sei nun Objektivität eingekehrt.

Bis 1966 war das Problem der Verkehrssicherheit politisch insofern wenig umkämpft, als für Verkehrsunfälle immer nur zwei erklärende Faktoren in Frage kamen: Fehlverhalten seitens der Verkehrsteilnehmer oder schadhafte Straßen. ${ }^{2}$ Mit der Kampagne von Nader trat ein dritter Faktor hinzu, und zwar die Autos selbst, die, wie damals festgestellt wurde, bei jeder Geschwindigkeit (und unabhängig von der Infrastruktur) unsicher sind. 40 Jahre später werben Autokonzerne mit Test-

1 Dieser Beitrag geht auf Literaturrecherchen und zwei Interviewserien mit Expertinnen und Experten zurück. Im Jahr 2004 habe ich im Rahmen eines vom deutschen Forschungsministerium (BMBF) finanzierten Forschungsprojekts (SMARTBENCH; TU Berlin) Leitungspersonen aus öffentlichen Forschungseinrichtungen, Ministerien und einem Automobilkonzern über Verkehrssicherheit als Objekt von Politik und Forschung in Frankreich befragt. Die zweite Serie ist datiert auf die Jahre 2009 und 2010 und umfasst Gespräche mit Leitungspersonen aus öffentlichen Forschungseinrichtungen, Verbänden und einem Automobilkonzern in Deutschland. Sie stand im Kontext eines von der französischen Agence Nationale de la Recherche (ANR) geförderten Forschungsprojekts zu Road Safety in European Governance (ROSARINE; INRETS).

2 Bardet 2008. 
ergebnissen aus dem »EuroNCAP «, einem Testverfahren, das sich neben den schon bestehenden etablieren konnte. Ist die enorme mediale Präsenz dieser Tests ein weiterer Beleg für den Aufstieg einer Auditgesellschaft, ${ }^{3}$ die durch » Rituale der Verifizierung " gekennzeichnet ist?

Die sozialwissenschaftliche Forschung zum Straßenverkehr hat sich in dieser Frage weitgehend festgelegt. Seit The culture of public problems ${ }^{4}$, einer Pionierstudie in diesem Feld, wurde immer wieder herausgestellt, dass Verkehrssicherheit eine Sache der Symbolpolitik ist. ${ }^{5}$ Auf der anderen Seite blieb offen, wie der Wandel zu erklären ist, dem dieses Feld im hier beobachteten Zeitraum unterlag.

Das Deutungsschema der Transformation des Politischen zur Symbolpolitik ${ }^{6}$ kann dabei jedoch nicht von der Agenda gestrichen werden. Es findet dort, wo öffentlich Zuständigkeit für ein Sicherheitsproblem reklamiert wird, einen Anwendungsfall von definitorisch klarer Abgrenzung. Wenn im Namen der Sicherheit symbolpolitische Maßnahmen ergriffen werden, so Thomas Birkland, dann hätten diese Aktionen in erster Linie das Ziel, sichtbar zu sein, was nicht immer gleichbedeutend mit "wirksam « sei. ${ }^{7}$ Im harmloseren Fall ist das eine Abwägungssache: Wenn unterschiedliche Sicherheitsmaßnahmen zur Wahl stehen, werde diejenige vorgezogen, die besonders viel Sichtbarkeit verspricht. In anderen Fällen bleibe keine Zeit, um unterschiedliche Maßnahmen abzuwägen. Der Ausnahmezustand, der zu Sicherheitsmaßnahmen ermächtigt, halte nicht lange an, und insbesondere wenn diese mit finanziellen Belastungen oder Freiheitsbeschränkungen verbunden sind, sinke rasch die Bereitschaft für neue Sicherheitsmaßnahmen. ${ }^{8}$

Hat Politik im Namen der Sicherheit also keine Wahl? Sind ihr hinsichtlich des Zeitpunkts (Maßnahmen sind unmittelbar nach großen Schocks zu ergreifen) oder hinsichtlich der Mittel (besonders sichtbare Maßnahmen sind vorzuziehen) die Hände gebunden? Das hieße allerdings, dass sich Sicherheitspolitik zwangsläufig

\section{Power 1997.}

4 Gusfield 1981.

5 Die zitierte Studie wurde unlängst anlässlich einer Neuausrichtung der Verkehrssicherheitspolitik in Frankreich wiederentdeckt (Cefaï 2009); in diesem Kontext genoss das Thema auch die bisher größte sozialwissenschaftliche Aufmerksamkeit (vgl. die Themenhefte von Espaces et sociétés 118/2004, Entreprises et histoire 51/2008 und Réseaux 147/2008). Soweit sie auf die aktuellen Maßnahmen eingehen, sehen die dort erschienenen Beiträge diese zu einseitig auf die Erfassung und Verfolgung von Verkehrsdelikten ausgerichtet (Gilbert 2008). Das Problem der Verkehrssicherheit werde auf diese Weise aus dem öffentlichen Raum verdrängt und stattdessen (wieder) als eine Frage des Umgangs mit abweichendem Verhalten gerahmt. Ungeachtet der Zahl der Verkehrstoten, die in Frankreich in den Jahren nach 2002 rückläufig ist, verdichtet sich also der Vorwurf, die neue verkehrspolitische Doktrin beschleunige einen Prozess symbolpolitischer Erosion. Die letzte Veröffentlichung in einer deutschsprachigen soziologischen Fachzeitschrift liegt fast 40 Jahre zurück und empfiehlt die Soziologie als eine »Hilfswissenschaft « der Verkehrssicherheitsforschung und -politik (Zelinka 1974).

6 Edelmann 1964.

7 Birkland 2004.

8 Ebd. 
und immer wieder diskreditieren muss. Denn stets lastet auf neuen Sicherheitsmaßnahmen der Verdacht, dass letztlich eine andere, verdeckte politische Agenda durchschlägt. Stets lässt sich jedoch auch umgekehrt fragen, wie real die Gefahren sind, die im Namen der Sicherheit »bekämpft « werden. Je öfter sich der Verdacht bestätigt, dass Unsicherheit und Sicherheit (nur) inszeniert werden, desto schwerer dürfte es sein, einen solchen Verdacht zu zerstreuen. Diese Umstände befördern ein beunruhigendes Deutungsmuster: Katastrophen führen geradewegs in einen Modus politischen Handelns, der als direkte Interessenverfolgung zu bezeichnen ist und an den vorgesehenen Instanzen und Verfahren vorbeiführt.

Im Umgang mit Extremsituationen hält Symbolpolitik Einzug ${ }^{9}$ und beschleunigt den Politikverlust. Übrig bleibt dann eine an Gesichtspunkten der Inszenierung ausgerichtete Politik ${ }^{10}$ und ihr Pendant: eine inszenierte »Phantom «-Öffentlichkeit. ${ }^{11}$ Es fällt jedoch auf, dass gerade Arbeiten, die diese Wirkungen der Symbolpolitik hervorheben, auch eine Gegenposition erkennen - angefangen bei Edelman, der neben einem "dramaturgischen Symbolwert " auch einen "politischen Nennwert " sieht, bis hin zu Sarcinelli12 ${ }^{12}$, der » regressiver " Symbolpolitik eine » reflexive « Variante gegenüberstellt. Unter letzterer versteht er ein kulturelles Repertoire oder einen "Zeichenvorrat ", dessen Pflege der politischen Kultur zugutekomme und von der kontextabhängigen Verwendung spezifischer Sets von Zeichen zu unterscheiden sei. ${ }^{13}$

Hier die Grundlagen (»politische Symbolik «), dort die Anwendung (»symbolische Politik «)? Diesen Vorschlägen haftet eine gewisse Verlegenheit an, denn es bleibt unbestimmt, wie sich symbolischer Vorrat und symbol(polit)ische Praxis zueinander verhalten. Solange dieses Problem der Arbitrarität auf eine Neuformulierung wartet, bleibt es bei der pauschalen Deutung fortlaufender Erosion: Symbolpolitik trete demnach an die Stelle von Politik. Je weiter dieser Prozess vorangeschritten ist, desto stärker ist der Blick auf Transformationen eingeschränkt. Es gibt kaum einen Grund, sich eingehend mit den tatsächlichen Arsenalen von (laufend erweiterten) Sicherheitskontrollen zu beschäftigen, weil immer schon feststeht, dass das Ende der Politik näher rückt; was sich nicht in diese Deutung fügt, wird zum »technischen Detail« erklärt oder übersehen.

Im Dickicht der Details, der Prüfungen und Audits verbergen sich jedoch interessante Wendungen, die auch im Fall der Straßenverkehrssicherheit mehr Aufmerksamkeit verlangen. Um das Jahr 1966 kommen erstmals bestimmte Testverfahren zum Einsatz, die es möglich machen, Autoherstellern nachzuweisen, dass sie versäumt haben, ihre Kundinnen und Kunden besser zu schützen. Entscheidend ist die Verlagerung der Aufmerksamkeit von der Straße ins Labor. In dem Maße, in dem per Laborversuch Autos und Komponenten auf die Auswirkungen von Zusammen-

9 Gilbert 1992.

10 Edelmann 1964.

11 Lippman 1925; vgl. Sarcinelli 2011.

12 Sarcinelli 2011, S. 147.

13 Ebd., S. 136. 
stößen (crashworthiness) hin untersucht wurden, haben die Unfälle auf der Straße ihren bloß akzidentiellen Charakter verloren. ${ }^{14}$ Die bisher zur Erklärung herangezogenen Faktoren »Verhalten « und »Infrastruktur " wurden im Test weitgehend neutralisiert, um eine bis dahin kaum betrachtete dritte Variable zu untersuchen. Statt von »Unfällen « (accidents), die es zu vermeiden gilt (»aktive Sicherheit «), ist dann auch von unvermeidbaren "Zusammenstößen " (crashes) die Rede, die als ein Problem »passiver Sicherheit " erscheinen. Mit dem von einem europäischen Forschungskonsortium entwickelten EuroNCAP etablierte sich ein Testverfahren, das bisher übliche Aufpralltests (crashtests) integriert und nicht allein die passive Sicherheit der vorderen Fahrzeuginsassen prüft, sondern auch die anderer (unter anderem auch nichtmotorisierter) Verkehrsteilnehmer berücksichtigt.

Diese Transformationen sind beträchtlich, bleiben aber in einer Perspektive, die einseitig auf Symbolpolitik abhebt, unsichtbar. Es bedarf also - und das kann auch aus der hier knapp umrissenen Genealogie dieses Konzepts geschlossen werden eines Gegengewichts. Um dieses auch lexikalisch kenntlich zu machen, werde ich von »Infrapolitik «sprechen.

Im sozialwissenschaftlichen Forschungsstand zur Verkehrssicherheit dominiert, wie gesagt, eine symbolpolitische Lesart, die Erklärungen dafür liefert, wie sich über reine Symbolpolitik bestehende Machtkonstellationen erhalten und stabilisieren. Was oberflächlich betrachtet nach einem politischen Prozess aussieht, ist demnach nur eine Inszenierung, die zu keinem Zeitpunkt Rückschlüsse auf das eigentliche politische Geschäft zulässt. Im Unterschied zu diesem Erklärungsschema zielen die folgenden Deutungen auf Wechsel und Wandel in Machtkonstellationen und in der betroffenen Praxis. Der Bezugspunkt ist dann nicht länger eine Organisation, die eine Unterscheidung von Sprechen und Handeln (talk und action) hervorbringt und sich darüber in den Rahmen einer bestehenden Machtkonstellation einnistet. Der Bezugspunkt für meine Deutungen sind dagegen Infrastrukturen des Prüfens. Wenn sich Formate und Verfahren des Prüfens verändern, dann lässt das Rückschlüsse auf gesellschaftliche Transformationsprozesse zu. ${ }^{15}$ Mit der Verschiebung der Bezugsgröße - von Organisationen und Symbolpolitik auf Prüfungen und Infrapolitik - ist sogar eine Neuinterpretation des Übergangs von der industriellen zur postindustriellen Gesellschaft gelungen. ${ }^{16}$

Um das Konzept der Infrapolitik zu entfalten und als alternative und komplementäre Erklärung kenntlich zu machen, bedarf es zunächst einiger konzeptueller Vorbemerkungen, die unter anderem bei der Soziologie der Kritik ansetzen (Abschnitt 2). Im Anschluss wird dieses Konzept am Beispiel der Verkehrssicherheit erprobt (Abschnitte 3 bis 5).

14 Leonardi 2010, S. 267.

15 Pinch 1993, S. $27 \mathrm{ff}$.

16 Boltanski, Chiapello 2003.

Leviathan, 40. Jg., 4/2012 


\section{Laborpragmatismus und Infrapolitik}

Aus symbolpolitischer Perspektive sind Prüfungen nur »Prüfungen «. Sie werden im politischen Prozess zitiert, sind aber lediglich lose mit ihm verbunden; sie ändern, so besagt es die herkömmliche Theorie der Symbolpolitik, nichts an den Interessenlagen und Machtkonstellationen, sondern stabilisieren diese allenfalls. Infrapolitisch betrachtet, liefern Prüfungen Formate und Instrumente für politische Prozesse; sie tragen darum auch maßgeblich zur Transformation des Politischen bei. Die Frage, wie diese Verbindung ausfällt, ob lose und symbolpolitisch oder fest und infrapolitisch, gewinnt in der Diskussion um die Ankunft der postindustriellen Wissensgesellschaft an Bedeutung. Daniel Bell ${ }^{17}$ neigte einer infrapolitischen Sichtweise zu. Mit dem Dritten Sektor boome auch das Prüfwesen. Als entscheidend für diese Entwicklung machte er einen bestimmten Wissenstyp aus, den er als »theoretisches Wissen " charakterisiert. Robert Reich ${ }^{18}$, Richard Florida ${ }^{19}$ und andere haben die Dominanz dieses Wissenstyps und die Bedeutung wissenschaftlicher Wissensproduktion relativiert. Statt durch einen (definierbaren) Wissenstyp sehen sie die soziale Ordnung postindustrieller Gesellschaften durch die Entstehung einer neuen Klasse von "Symbolanalytikern « bestimmt. ${ }^{20}$ Deren Kompetenz liege nicht in Theoriearbeit und Wissenserzeugung, sondern in der "Manipulation " von Zeichensystemen. Diese Sicht auf »Wissensarbeit" steht einer symbolpolitischen Lesart nahe. Im Zusammenhang mit technischen Prüfungen greifen beide Perspektiven meines Erachtens etwas zu kurz. Einerseits genügt es nicht, allgemein auf »theoretisches Wissen « abzustellen (Bell), denn es geht hier um konkrete Wissensbestände, die auf Laborsituationen und -aufbauten bezogen sind. Andererseits beschränkt sich die Wissensarbeit, die im Zusammenhang mit Prüfungen anfällt, nicht auf Symbolmanipulation (Reich, Florida).

Der Fokus auf laborförmige Prüfungen geht auch über das Konzept der Subpolitik hinaus, das Ulrich Beck ${ }^{21}$ im Zusammenhang mit technischen und wissenschaftlichen Risiken zur Diskussion gestellt hat. Subpolitik steht bei ihm für politische Initiativen, die außerhalb staatlicher Institutionen und häufig in Opposition zu diesen entstehen: hier die »Aktionsleere der politischen Institutionen «, dort die »institutionenlose Renaissance des Politischen «. ${ }^{22}$ In dieser Gegenüberstellung von »Institutionen « und »Individuen « bleibt die Frage nach Praxisformen dieser Subpolitik allerdings ausgespart. Es finden sich allenfalls Hinweise darauf, dass sie überaus »beweglich « ist - vor allem im Kontrast zur »Unbeweglichkeit des staatlichen Apparates «. ${ }^{23}$ Die Diagnose von der »Individualisierung politischer Konflikte und

17 Bell 1973.

18 Reich 1992.

19 Florida 2002.

20 Vgl. auch Koppetsch 2011.

21 Beck 1993, S. $154 \mathrm{ff}$.

22 Ebd., S. 155.

23 Ebd., S. 156. 
Interessen $^{24}$ muss darum als überaus vorläufig betrachtet und möglicherweise revidiert und erweitert werden. ${ }^{25}$

Thesen zur symbolpolitischen Transformation des Politischen werden seit langem diskutiert und sind weit ausgereift (siehe Einleitung). Sie rufen nach einem infrapolitischen Gegenstück, das noch der Ausarbeitung bedarf. Dafür ziehe ich die Soziologie der Kritik und (darüber vermittelt) die laborethnografische Wissenschaftsforschung heran, weil diese konzeptuell wie empirisch einen Bezugspunkt offenlegen: Prüfungen sind ein Scharnier, um das Verhältnis von Infrapolitik und Politik zu klären; anhand von Prüfungen lässt sich das Verhältnis von Symbolpolitik und Infrapolitik empirisch untersuchen.

Für sich genommen, sind die genannten Quellen inzwischen sehr gut eingeführt, zum einen unter dem Etikett des »Laborkonstruktivismus «26, zum anderen auch unter dem Titel "pragmatische Soziologie « 27 . Sie weisen Familienähnlichkeiten auf ${ }^{28}$ und haben Gemeinsamkeiten, die sich für den Kontext der hier behandelten Problemstellung nutzbar machen lassen. Eine Kombination dieser beiden Ansätze, die auch den Neologismus »Laborpragmatismus « rechtfertigt, soll einige Probleme und Schieflagen überwinden, die dabei entstanden sind, die Rolle laboratorischer und laborförmiger Prüfung in der Gesellschaft theoretisch und empirisch zu erschließen. Bisher verteilt sich die Aufmerksamkeit für Laborgrenzen asymmetrisch: Die Laboratorisierung wird bisher vornehmlich nur in einer Richtung beobachtet: laboreinwärts. Das heißt, man beobachtet, was im Labor oder in laborähnlichen Situationen wie zum Gegenstand der Prüfung wird. Wenn das Augenmerk doch auf den Verkehr laborauswärts gerichtet wird, dann geschieht dies unter der sehr allgemeinen Annahme, dass die Laboratorisierung des Sozialen ein kaum aufzuhaltender, hoch dynamischer und expansiver Vorgang ist. Schrumpfen, also De-Laboratorisierung ${ }^{29}$, wird dagegen fast nie beachtet. Je stärker nun die Pragmatik des Prüfens betont wird und damit hervortritt, desto mehr verschwimmt die Grenze und die Differenz zwischen Labor und Gesellschaft - und umgekehrt. Um einem soliden Konzept von Infrapolitik näher zu kommen, sind diese Probleme zu klären und, soweit möglich, zu lösen.

Bruno Latour versteht unter Prüfungen Realitätsprüfungen: » Real ist, was sich in einer Prüfung als Widerstand erweist « ${ }^{30}$ In dieser elementaren, quasi physikalischen Bestimmung geht es um "Kräfte", die mit Hilfe von Prüfungen isoliert und als isoliert darstellbar gemacht werden. Dazu bedürfe es einer raffinierten materiellen Struktur und ausgefeilter Praktiken; diesen Vorrichtungen (Dispositiven) des Beobachtens und Messens gilt die Aufmerksamkeit ethnografischer »Laborstu-

24 Ebd., S. 161.

25 Vgl. de Vries 2007; Linhardt, Muniesa 2011.

26 Vgl. unter anderem Latour, Woolgar 1986.

27 Breviglieri et al. 2009.

28 Guggenheim, Potthast 2012.

29 Guggenheim 2012.

30 Latour 1984, S. 177.

Leviathan, 40. Jg., 4/2012 
dien «. Eine große Zahl von Fallstudien hat derartige Prozesse der Laboratorisierung im engeren, also innerwissenschaftlichen Sinn untersucht. Immer wieder wurden Erkenntnisverfahren und ihre Transformation aus größter Nähe betrachtet. Seit den Pionierstudien ${ }^{31}$ wurde dieses Interesse an der »Wissensproduktion « als ein entscheidender Fortschritt gegenüber wissenschaftssoziologischen Ansätzen angesehen, die sich bis dahin weitgehend auf den institutionellen Kontext wissenschaftlicher Produktionstätigkeiten beschränkt haben. In Absetzung und Ergänzung zur institutionalistischen Wissenschaftssoziologie wurde gezeigt, dass und wie Untersuchungsgegenstände »konstruiert ", das heißt in manipulierbare epistemische Objekte verwandelt werden. ${ }^{32}$ Die Wissenschaftsforschung verfügt inzwischen über einen ausgedehnten, allerdings sperrigen Wissensfundus; sie kann zeigen, wie sich Mechanismen, Verfahren und Methodologien des Messens und Prüfens etablieren, die bis dahin unsichtbare oder flüchtige Gegenstände verfügbar und kontrollierbar machen. Sie rekonstruiert, wie mit manchmal gehörigem apparativen Aufwand Untersuchungsgegenstände zerlegt, verkleinert und vergrößert wurden, um dann unabhängig von räumlichen und zeitlichen Einschränkungen auf kontrollierte Weise handhabbar werden zu können. Mit der Ankunft solcher Art von Prüfungen hat sich das Gefüge der naturwissenschaftlichen Disziplinen stark verändert. Disziplinen, die Laboratorisierungsschübe durchlaufen haben, haben gegenüber Nachbardisziplinen, denen dies vorenthalten blieb und die sich weiterhin am Paradigma der Feldforschung orientieren, institutionell betrachtet, einen rasanten Aufschwung genossen. ${ }^{33}$

Die innerwissenschaftliche Dynamik, die von diesen Prozessen der Laboratorisierung ausging, schlug sich unter anderem in anwachsenden Geräteparks, immer weiter ausgefeilten Messtechnologien und zunehmend umfangreichen Infrastrukturen nieder. Sie hat hier und dort zu Erscheinungen des akademischen Kapitalismus und von Big Science geführt. Daraus erwuchs die Frage nach den außerwissenschaftlichen Umständen und Folgen dieser Laboratorisierungen - eine Frage, die die von Bruno Latour und anderen lancierte Theorie der Akteursnetzwerke in der Folge auf besonders konsequente Weise verfolgt hat. Sie sieht Laborprüfungen nicht auf einen innerwissenschaftlichen Kontext beschränkt, sondern macht sich zur Aufgabe, die aus ihren Zusammenhängen herausgelösten Prüfungen und ihre »Rekontextuierung « in anderen gesellschaftlichen Zusammenhängen zu untersuchen. ${ }^{34}$ Allerdings verzichtet sie dabei auf einen Gesellschaftsbegriff, der demjenigen der den Laboratorien entwachsenden Laborprüfungen entgegengesetzt werden könnte. Gesellschaft fällt zusammen mit Prozessen der vergesellschaftenden Laboratorisierung. Die Theorie der Akteursnetzwerke trifft keine begrifflichen Vorbestimmungen über

31 Siehe beispielsweise Latour, Woolgar 1986.

32 Für einen Überblick vgl. Heintz 1998.

33 Zur Unterscheidung von labor- und feldwissenschaftlichen Disziplinen vgl. Kohler 2002. Das jüngste und prominenteste Beispiel für Laboratorisierung ist die Molekularbiologie; vgl. Rabinow 1996.

34 Latour 1984. 
die Qualität dessen, was durch dieses oder jenes, mehr oder weniger ausgedehnte Akteursnetzwerk überschrieben wird.

Genau dieser Punkt stand auch im Zentrum der Kritik. Der Verzicht auf einen Gesellschaftsbegriff sei unzulässig; wer das Verhältnis von Labor und Gesellschaft nicht begrifflich fixiere, mache sich einer unterkomplexen Darstellung schuldig und könne nur mit krachenden »Implosionen « oder »Explosionen « aufwarten: ${ }^{35} \mathrm{Mal}$ schrumpfen Gesellschaften auf Laborgröße, mal blähen sich Experimente auf und sprengen die Laborgrenzen. Die Betonung der zentralen Rolle des Labors für die Gesellschaft galt (auch den zitierten) Vertretern institutionalistischer Wissenschaftssoziologie allerdings schon vorher als wenig theoriefähig. Sie hatten nach Tschernobyl eindringlich vor einer Ineinssetzung von Gesellschaft und Labor gewarnt: Was an der Tschernobyl-Konstellation, dem "größten anzunehmenden Experiment «36, und einer "Gesellschaft als Labor « ${ }^{37}$ politisch bedrohlich erscheinen musste, blieb de facto auch theoretisch undenkbar. Mit jedem Verweis auf Tschernobyl wuchs die Gewissheit, dass die Laboratorisierung der Gesellschaft nichts mit Prüfungen zu tun hat - und wenn doch, dann mit ihrem vollständigen Versagen. Forschungen zur Frage der Rolle des Labors in der Gesellschaft kamen möglicherweise auch aus diesem Grund nicht so recht voran und wurden über längere Zeit dort übersehen, wo sie längst schon wieder aufgenommen und zur Revision vorgelegt worden waren.

Ein solcher Ort sind die Arbeiten von Luc Boltanski und Laurent Thévenot. ${ }^{38}$ Dort wird eine Perspektive auf Prozesse der Kritik und der Rechtfertigung entwickelt, die sich am Modell der Laborprüfung orientiert. Zugleich wird die Frage nach dem Schrumpfen oder Wachsen von Laborgrenzen als eine Frage der »Größe« reformuliert. »Groß « ist, was sich in einer laborartigen Prüfung als Gemeinwohl bewähren konnte. Prüfungen sind jedoch nicht auf eine einzige Ordnung des Gemeinwohls geeicht; vielmehr führen die Autoren unter Rückgriff auf prominente Entwürfe aus der politischen Philosophie mehrere Begründungen des Gemeinwohls ein, die dann konsequent "symmetrisch " behandelt werden. Auf diese Weise wird ein vielfältiges Verständnis von Prüfungen (auf Gemeinwohl) bereitgestellt. »Prüfen « heißt dann nicht mehr nur: in einen Zustand der Messbarkeit überführen (Latour), sondern auch: im Namen unterschiedlicher Ordnungen des Gemeinwohls rechtfertigen.

Boltanski und Thévenot zeigen sich an einer Integrationsleistung interessiert. Sie bringen Latours Aufmerksamkeit für die Pragmatik von Laborprüfungen mit einer Pluralität von Rechtfertigungsordnungen zusammen. Prüfungen beruhen demnach auf Akten der generalisierenden Zuordnung partikularer Sachverhalte zu allgemein gültigen Prinzipien des Gemeinwohls, die, wenn sie angefochten werden, zu wiederholen sind. Darüber entsteht eine auf Prüfungen drängende Dynamik, die Rechtfertigungen und Explikationsleistungen hervorbringt und so lange von anderen und unmittelbaren Handlungszwängen entlastet. Im Unterschied zu Arbeiten, die sich

35 Hasse et al. 1994, S 246, 249.

36 Weingart, Krohn 1986.

37 Krohn, Weyer 1990.

38 Boltanski, Thévenot 2007.

Leviathan, 40. Jg., 4/2012 
ausschließlich an der Theorie der Akteursnetzwerke orientieren, vermittelt diese Ausarbeitung also eine deutliche Vorstellung davon, was Infrapolitik eigentlich politisch bedeutet. Wie Boltanski selbst notiert, beobachte Latour "Prüfungen" unter dem Aspekt des »Kräftemessens " (épreuves de force), während er in Zusammenarbeit mit Laurent Thévenot einen Begriff der Prüfungen der »Größe« ausgearbeitet habe (épreuves de grandeur). Spätestens seit der Studie zum Neuen Geist des Kapitalismus ${ }^{39}$ liegt zudem ein Vorschlag vor, dies als eine begriffliche Dopplung zu nutzen: Um zu erklären, wie eine vormals wirksame Kapitalismuskritik, aus der ein Geflecht hoch institutionalisierter Prüfungen hervorgegangen ist, binnen kurzer Zeit entkräftet wurde, rekonstruieren Boltanski und Chiapello »Ausweichmanöver «, bei denen Prüfungen auf Größe (erkämpfte und laufend geprüfte Sozialstandards) zugunsten von bloßen »Kraftproben « (Verlegungen von Produktionsstandorten) umgangen werden.

Im Alltagsverständnis von Sicherheitsproblemen gibt es ein wiederkehrendes Motiv: Sicherheitsprobleme sträuben sich gegen territoriale Einhegungen. Wenn ein Sicherheitsproblem lokal gelöst ist, tritt es an anderer Stelle wieder auf. Für diese Möglichkeit der Verlagerung finden sich im Bereich der Straßenverkehrssicherheit Beispiele in großer Zahl. ${ }^{40}$ Zudem hat sich mehrfach die Instanz geändert, die über Risiken befindet und damit Verantwortlichkeiten verteilt und »umverteilt « ${ }^{41}$ Prüfungen auf passive Sicherheit wurden von der Straße ins Labor und von dort in mathematische Modelle verlagert. ${ }^{42}$ In den folgenden Abschnitten wird eine ähnliche Wanderung rekonstruiert: Wie und an welchen Orten werden Risiken und Probleme der Verkehrssicherheit geprüft? Welche Kategorisierungen kommen dabei zum Tragen? Dieser Fokus auf Prüfungen empfiehlt sich auch als ein forschungsökonomisches Prinzip: Laboratorien (von DEKRA und TÜV bis hin zu öffentlichen und privaten Forschungseinrichtungen) sind auch in diesem Fall Orte der »Verdichtung " von Gesellschaft ${ }^{43}$ und deshalb sorgfältig zu analysieren.

In einer laborpragmatisch geschulten infrapolitischen Sichtweise auf Verkehrssicherheit zeigt sich eine Fülle verschiedener Prüfformate. Wenn es gelingt, ein historisches Verständnis des Wachstums und der Entwicklung von Dispositiven der Prüfung auf analytische Unterscheidungen abzustützen, dann lässt sich einschätzen, inwiefern symbolpolitische Maßnahmen auch eine Infrapolitik haben. Folgen neue Auflagen den Vorgaben der bestehenden Sicherheits- und Prüfarchitekturen oder

39 Boltanski, Chiapello 2003.

40 Ein streitbares Beispiel für dieses Phänomen der Risikoverlagerung liefert John Adams (1985). Es sei zweifelhaft, dass die Einführung der Gurtpflicht zu einem Gewinn an Verkehrssicherheit geführt habe, denn alle öffentlichen Verlautbarungen in dieser Sache beruhten auf einer äußerst dezentralen und darum wenig verlässlichen Berichtspraxis. Zahlen über Verkehrstote könnten als sicher durchgehen. Gegenkontrollen jedoch, ob sich das Unfallgeschehen in schwerer erfassbare Kategorien und an Orte verlagert hat, an denen die Dunkelziffer besonders hoch ist, seien jedoch nicht möglich.

41 Wetmore 2004.

42 Leonardi 2010.

43 Knorr Cetina 1988. 
brechen sie mit ihnen? Wie schwer wiegt der Bestand gegenüber neu getroffenen Maßnahmen und zwingt - wie etwa im Beispiel der Terrorismusprävention an Flughäfen - zu einer inkrementellen Vorgehensweise? ${ }^{44}$

\section{Eine Karriere für die Fahrzeugsicherheit}

Der Materialbestand, auf den die Analyse zugreift, erlaubt es, mich einer Vorentscheidung für eine symbol- oder infrapolitische Deutung zu enthalten. Er bietet einerseits reichhaltige Einblicke in mehr oder weniger lokale und disparate Praktiken des Testens und Prüfens; auf der anderen Seite enthält er eine sehr geschlossene Darstellung, die von Kontingenzen absieht und darauf bedacht ist, Einheit und Kontinuität herauszustellen. Beides findet sich nebeneinander und unvermittelt in der ausführlichen Karriereerzählung von Herrn S., ein Ingenieur, der ab 1968 knapp 35 Jahre lang in der Automobilindustrie gearbeitet hat und durchgehend mit Sicherheitsfragen befasst war. Ich werde seine Ausführungen zunächst biografisch verdichten: als eine Folge von Ereignissen, die sich im Rückblick als persönliche Herausforderungen darstellen. Dabei wird eine Periodisierung erkennbar, die zumindest für deutsche Autohersteller typisch und gut belegt ist. ${ }^{45}$ Darum lassen sich das so vorstrukturierte berufsbiografische Material und die geschilderten Prüfungen im Rahmen einer symbolpolitischen (4) und einer infrapolitischen Interpretation (5) als Pars pro toto für die allgemeine Entwicklung der Verkehrssicherheit betrachten. ${ }^{46}$

\subsection{Marktzugang}

Infolge der erwähnten Verschärfungen der Sicherheitsauflagen für den Zugang zum US-Markt (1966) wechselt Herr S., beschäftigt bei einem Autohersteller und Spezialist für "Vorschriften ", zum letzten Mal den Arbeitgeber. Er wird von einem Konkurrenzunternehmen abgeworben und kann sich dabei beruflich verbessern. Als Leiter der Vorschriftenabteilung begleitet er auch seinen neuen Arbeitgeber dabei,

44 Vgl. Potthast 2011.

45 Stieniczka 2006.

46 Die meisten der in der ersten Fußnote genannten Experteninterviews hatten zur Bedingung, dass ich Anonymisierung zugesichert und auf eine Ton-Aufzeichnung verzichtet habe. Das gilt auch für das rund dreistündige und in seiner Materialtiefe singuläre Gespräch mit Herrn S., das hier im Zentrum steht. Kontakte und Zugänge, über die ich Herrn S. identifiziert habe, verdanke ich unter anderem dem Umstand, dass ich für Anfragen eine renommierte französische Institutsadresse verwenden konnte. Ich habe Herrn S. in der Folge eine vorläufige Interpretation (Potthast 2012) zugeschickt und postwendend einen Kommentar erhalten: "Zu Ihrem Beitrag muss ich sagen, dass ich [...] wohl selten jemanden erlebt [habe], der mir so lange zugehört und davon auch noch so viel mitgenommen hat. Kompliment! « Die diesem Lob folgende ausführliche Rückmeldung ist in den vorliegenden Text eingegangen. Eine methodische Faustregel hat sich also erneut bestätigt: In schwer zugänglichen Feldern sind Pensionäre eine wichtige Quelle: Sie haben mehr Zeit und tun sich leichter, als reflektierte Praktiker aufzutreten (Kompliment zurück!). 
die strengeren Sicherheitsvorschriften des US-Marktes für die Fahrzeugentwicklung insgesamt verbindlich zu machen. Der Vorschriftenabteilung hat dies im Gefüge des Konzerns zu bis dahin undenkbarer Sichtbarkeit verholfen.

\subsection{Schließtechnik}

So erklärt sich Herr S., dass die Konzernleitung ausgerechnet ihm wenig später eine Aufgabe überträgt, für die er eigentlich weder qualifiziert noch zuständig ist. Sie besteht darin, umgehend auf eine anhaltende Diebstahlserie zu reagieren. Unter anderem hatte ein ausländisches Versicherungsunternehmen angedroht, Fahrzeugtypen, die weiterhin durch hohe Diebstahlquoten auffallen, vom Versicherungsschutz auszuschließen. Es sei ihm gelungen, diese Aufgabe zu lösen, obwohl sie, wie für Sicherheitsprobleme typisch, unvorhersehbar und jenseits abgesteckter technischer Zuständigkeitsbereiche aufgetreten war und eine rasche und flexible Koordination quer zu etablierten Abteilungsgrenzen verlangt habe.

\subsection{Unfallforschung}

Wiederum wenig später, ab Mitte der 1970er Jahre, wird Verkehrssicherheit in Deutschland und anderen westeuropäischen Ländern auf politische Initiative hin zu einem Gegenstand öffentlich geförderter, zunächst stark interdisziplinär ausgerichteter Forschung. Herr S. beschreibt am Beispiel der »interdisziplinären Unfallforschung ", wie sich sein Tätigkeitsgebiet verwissenschaftlicht und darüber neu strukturiert. Als sich der Konzern entscheidet, in Kooperation mit öffentlichen Forschungseinrichtungen und Kliniken Unfallforschung zu betreiben, wird diese Kompetenz in der Vorschriftenabteilung angesiedelt und damit der Leitung durch Herrn S. unterstellt.

\section{4 Öffentlichkeitsarbeit}

Gegen Ende der 1980er Jahre entfaltet sich in Deutschland eine Debatte, in der Autos als Umweltsünder dargestellt werden. Die Konzernleitung nimmt diese Debatte ernst und reagiert offensiv. Sie überträgt Herrn S. einen Forschungsauftrag, der nicht mehr in das Format der Unfallforschung passt. Er ist nunmehr »Beauftragter des Vorstands « für alle Themen, die mit »Politik « zu tun haben. In seiner neuen Funktion reist er nach eigener Darstellung von Podium zu Podium und eilt von Interview zu Interview. Diese Episode hat Herrn S. eine direkte Verbindung zum Konzernvorstand beschert und markiert den Höhepunkt seiner Karriere.

\subsection{Verbraucherinformation}

In den 1990er Jahren konstituiert sich zum Thema Fahrzeugsicherheit ein europäisches Forschungskonsortium, das in der Folge bei den Akteuren dieses Felds auf außergewöhnliche Beachtung stößt. Wie eingangs erwähnt, werden unter dem Akronym EuroNCAP nach einem gegenüber bestehenden Testverfahren eigenständigen Drehbuch Testserien zur inneren und äußeren Sicherheit durchgeführt. Herr S. berichtet, dass diese Umstellung im Konzern Turbulenzen ausgelöst hat. Warum 
sollte ein Autohersteller, der Sicherheit selbstverständlich exklusiv den Fahrzeuginsassen auf den Vordersitzen zukommen ließ, nun für die Sicherheit der anderen zuständig sein? Als er seine Rente antritt, ist die unternehmensinterne Debatte, wie man sich zum EuroNCAP verhalten soll, noch nicht abgeschlossen.

\section{Eine symbolpolitische Deutung}

Ihren Ausgangspunkt nimmt die Entwicklung in den USA, wo sich Mitte der 1960er Jahre eine Umdeutung der Risiken des Straßenverkehrs erkennen lässt, die sich rasch auch in Europa und in der Karriere von Herrn S. bemerkbar macht. Diese und die darauf folgenden, hier im Überblick gezeigten Karrierestationen enthalten jeweils eine symbolpolitische Lektion, die nun Abschnitt für Abschnitt herausgearbeitet wird.

\begin{tabular}{|l|c|c|}
\hline Karrierestation & Symbolpolitik? & Infrapolitik? \\
\hline 3.1 Marktzugang & 4.1 & 5.1 \\
\hline 3.2 Schließtechnik & 4.2 & 5.2 \\
\hline 3.3 Unfallforschung & 4.3 & 5.3 \\
\hline 3.4 Öffentlichkeitsarbeit & 4.4 & 5.4 \\
\hline 3.5 Verbraucherinformation & 4.5 & 5.5 \\
\hline
\end{tabular}

\subsection{Sicherheit und re-inszenierte Technikgeschichte}

Obwohl Konsens darüber besteht, dass es eines externen Anstoßes bedurft hatte, um die Entwicklung sicherer Fahrzeuge in Gang zu setzen, geben sich die Automobilhersteller die größte Mühe, die Geschichte der Fahrzeugsicherheit fortlaufend nach ihren Maßstäben zu revidieren. Herr S. beteiligte sich engagiert an dieser Revision. Fahrzeugsicherheit sei eine Erfindung der Hersteller, ihre Entwicklung habe sehr früh begonnen und sei konsequent und geradlinig fortgeschrieben worden. Damit verfällt er in eine Darstellung, die den Beitrag von Akteuren außerhalb der Automobilkonzerne stark unterschätzt und die Geschlossenheit und Rationalität ingenieurtechnischen Wirkens innerhalb der Unternehmen überschätzt. Die Vorstellung, dass die Verbraucherkampagne, die Mitte der 1960er Jahre in den USA gegen unsichere Autos lanciert wird, als eine dramatische "soziale Öffnung " des »Dispositivs der (passiven) Fahrzeugsicherheit « gelten kann, ${ }^{47}$ ist damit nicht vereinbar. Die Unstimmigkeiten, die die hagiografischen Revisionen zur Geschichte der passiven Sicherheit erzeugt haben, sind gut belegt. ${ }^{48}$

Wenn Herr S. die Anfänge der Fahrzeugsicherheit zurückdatiert (das erste Patent auf Insassenschutz durch stabile Fahrgastzelle und Knautschzone, Vollschutzrah-

47 Stieniczka 2006.

48 Ebd. 
men, Sicherheitsgurte, Scheibenbremse etc.), dann ist das insofern bemerkenswert, als er seine eigene Beteiligung schwächt und seine biografische Erzählung unterminiert. Die Vorschriftenabteilung sieht sich in dieser Version wieder degradiert. Es spielt keine Rolle mehr, ob es dieser Abteilung gelingt, sich abzeichnende Vorgaben $\mathrm{zu}$ antizipieren und dadurch zur Initiatorin für Wettbewerbsvorteile zu werden, wenn die genannten Innovationen der Fahrzeugsicherheit der Entwicklungsabteilung zuzuschreiben sind und sich angeblich aus der Logik des technischen Fortschritts ergeben. Es ist unschwer zu erkennen, dass hier eine symbolpolitische Investition vorgenommen wird, die darauf zielt, eine erfolgreiche verbraucherpolitische Kampagne (Unsafe at any speed), die die Autolobby seinerzeit überrumpelt hat, ihrerseits zu einer symbolpolitischen Operation zu erklären.

\subsection{Sicherheitstechnische Lösung für ein wohl inszeniertes Problem}

Die Reaktion auf den drohenden Verlust des Versicherungsschutzes folgt einem Muster, das für sicherheitstechnische Innovationen typisch und bekannt ist. War die Schließtechnik der alten Modelle das Problem, dann ist die überarbeitete Schließtechnik die Lösung. Zugleich gibt Herr S. zu verstehen, dass es mit einer technischen Problembearbeitung nicht getan war. Er schildert, wie es ihm auf unorthodoxe Weise gelungen ist, aus einem drängenden, seine Kompetenz überschreitenden Problem sogar noch einen Medien-Coup zu machen: In Zusammenarbeit mit dem LKA habe er einen inhaftierten Autodieb bei laufender Kamera und Stoppuhr aufgefordert, ein entsprechend nachgerüstetes Fahrzeug aufzubrechen. Dieser Nachweis für symbolpolitische Kompetenz habe seine Karriere befördert.

\subsection{Wohl inszenierte Forschung für technische Lösungen}

Nach Darstellung von Herrn S. haben die Autohersteller rasch verinnerlicht, Sicherheitskomponenten nicht nur auf Vorschrift nachzurüsten. Die Lage habe sich umgekehrt: Autokonzerne entwickeln laufend neue technische Lösungen, die sie als der Sicherheit förderlich einstufen; die Herausforderung bestehe nun vielmehr darin, die Prüfbehörden, die die Zulassung überwachen, von deren Nutzen zu überzeugen.

Dieses Problem stelle sich weniger bei Vorrichtungen, die der passiven Sicherheit zugutekommen. Deren Wirkung könne per Crashtest zwar mit viel Aufwand, aber mit augenscheinlicher Evidenz nachgewiesen werden. Schwieriger ist das bei Maßnahmen, die zur Steigerung der aktiven Sicherheit beitragen sollen. Hierzu hat sich nach Überzeugung von Herrn S. die detaillierte Beschäftigung mit Unfallverläufen als nützlich erwiesen. Das prominenteste Beispiel, das er als Beleg anführen kann, ist das Anti-Blockier-System (ABS). Erst als die Konzernforscher bei den Präsentationen im Zusammenhang mit seiner Einführung auf den Anteil und die Verlaufsformen von Schleuderunfällen verweisen konnten, habe man die Prüfer, von Haus aus überwiegend Juristen, beeindruckt: »Vier parallele Bremsspuren! [Wie soll ein Fahrzeug mit vier blockierenden Rädern noch manövrierbar sein?] «

Herr S. berichtet, dass er diese Argumente auch firmenintern geltend gemacht habe. Es sei sinnvoll gewesen, sich an Kooperationen für Unfallforschung zu beteiligen; und es sei legitim, den Kundinnen und Kunden die Kosten dafür in Rechnung 
zu stellen. Herr S. kommt immer wieder darauf zurück, dass das Personal bei den prüfenden Behörden meistens "nicht vom Fach « ist. Wenn er also empfiehlt, auf Forschung und Wissenschaft zu setzen, dann sucht er nicht den Dialog mit der Fachöffentlichkeit. Dies würde ihm den Ruf einbringen, Glasperlenspiele zu veranstalten. Es ist vielmehr so, dass Wissenschaft ausdrücklich dafür eingesetzt wird, um - mit auf geeignete Weise aufbereiteten Ergebnissen - Laien zu beeindrucken. Das lässt sich als eine symbolpolitische Vereinnahmung der Unfallforschung bezeichnen.

\subsection{Re-Delegierung}

Innerhalb des Konzerns lässt sich über die geschilderten Episoden hinweg von einem allmählichen Aufstieg des Themas Sicherheit sprechen: von der Vorschriftenabteilung zur sicherheitstechnischen Innovation und weiter zur Unfallforschung mit externen Partnern. In der vierten Episode gerät das Thema »Sicherheit « unmittelbar in Tuchfühlung mit der Konzernspitze. Damit reagiert der Konzern auf eine Protestmobilisierung, die sich gegen Autos wendet. Herr S. erhält einen diffusen Forschungsauftrag, der vor allem darin besteht, bei medienwirksamen Veranstaltungen als » Experte « aufzutreten oder, mit seinen Worten, für seinen Arbeitgeber als »Prügelknabe « herzuhalten. Hier ist Sicherheit weitgehend, wenn nicht vollständig von konkreten, technischen Entwicklungsaufgaben entkoppelt und in diesem Sinn eine ganz überwiegend symbolpolitische Aktivität. Es geht in erster Linie um mediale Präsenz, also vor allem darum, den autokritischen Expertinnen und Experten nicht das Feld zu überlassen, sondern ihre Positionen - mit Bezug auf Wissenschaft systematisch zu hinterfragen und zu relativieren.

Herr S. hat diese Haltung bewahrt. Auch nach seiner Pensionierung achtet er stets darauf, dass die Verantwortung für Risiken des Straßenverkehrs nicht allein den Fahrzeugen und den Herstellern zugeschrieben wird. Die Absicht, eine symbolpolitische Beweisführung zu kontern und die Verantwortung zurückzudelegieren, äußert sich unter anderem in terminologischer Wachsamkeit. Wie viele andere in diesem Feld ermahnt er mich, begrifflich präzise zu sein und »Verkehrssicherheit " (auch in einem Gespräch, dessen Kontext darüber kein Missverständnis zulässt) nicht mit »Fahrzeugsicherheit " gleichzusetzen.

\subsection{Die Inszenierung mechanischer Objektivität}

Mit der letzten Episode, in der sich die Autohersteller mit dem EuroNCAP und der unvorhersehbaren Dynamik dieses verbraucherpolitischen Instruments auseinandersetzen müssen, gelangen Aufpralltests mit medialer Unterstützung zu größter Prominenz. Herr S. äußert sich in diesem Zusammenhang defensiv: Auch der EuroNCAP müsse die langen Entwicklungszyklen für neue Modellreihen respektieren.

\subsection{Zwischenfazit}

Im Straßenverkehr gibt es nur selten spektakuläre Unfälle, die die Medien über die lokale Berichterstattung hinaus und über längere Zeit beschäftigen. Der letzte Ver- 
kehrsunfall, der es in Deutschland zu einem Medienereignis gebracht hat, geht auf das Jahr 2004 zurück. Ein Daimler-Testfahrer stand unter Verdacht, mit derart hohem Tempo unterwegs gewesen zu sein, dass die Fahrerin eines Kleinwagens vor ihm überstürzt die Spur geräumt und in der Folge mit ihrem Kind den Tod gefunden hat. ${ }^{49}$ Dieser Fall bot Angriffspunkte zur Skandalisierung: Sind Deutschlands Straßen öffentliche Rechtsräume - oder Versuchsanlagen für die übermotorisierten Fahrzeuge mächtiger Konzerne? Versäumen Staat und Ordnungskräfte ihre Schutzpflicht?

Auch die hier betrachteten Schlüsselepisoden sind »Medienereignisse ", aber sie handeln nicht von gefährlichen Testfahrten jenseits des Versuchsgeländes, sondern von wohl kontrollierten und strategisch inszenierten Praktiken des Testens.

In den Episoden lässt sich ein wiederkehrendes Muster erkennen: Jede neue Prüfung, die den Autoherstellern von außen aufgezwungen wird, mutiert binnen kurzer Zeit zu einer Plattform für symbolpolitische Aktivitäten. Dies erweckt den Eindruck, dass sich das Geschehen immer wieder neu auf eine symbolpolitische Bühne verlegt und dass Inszenierungen und Gegeninszenierungen in gewisser Weise fast den Charakter einer in sich geschlossenen Veranstaltung annehmen. Die Aufmerksamkeit der Beteiligten gilt zunehmend und in der vierten Episode (»Podium «) ausschließlich einem verwickelten Spiel der Abwehr und Weitergabe von Schuldzuweisungen (blame $\left.\operatorname{game}^{50}\right)$. Insoweit findet sich der sozialwissenschaftliche Forschungsstand bestärkt, der im Fall der Verkehrssicherheit überwiegend auf symbolpolitische Erklärungsmuster gesetzt hat.

Längst hat sich der symbolpolitische Vorbehalt dahingehend radikalisiert, auch vor wissenschaftlich gestützten Prüfungen keinen Halt zu machen. Die Ausführungen von Herrn S. geben Aufschluss darüber, wie Verkehrssicherheit zum Gegenstand von Forschung und Wissenschaft geworden ist: keineswegs mit der Folge, dass das symbolpolitische Spiel ein Ende genommen hätte. Die Vorstellung, dass die Wissenschaft die Öffentlichkeit mit neutralen Problemformulierungen beliefert, erscheint stark ergänzungsbedürftig. Statt Wissenschaft als eine geschlossene Maschine zu betrachten, die Definitionen für öffentliche Problemlagen auswirft, ist darum schrittweise nachvollziehbar zu machen, wie über infrapolitische Prozesse Öffentlichkeit hergestellt wird. Wie oben angemerkt, neigen auch die Laborstudien, die für die infrapolitische Perspektive Pate stehen, dazu, die Rolle der Wissenschaft zu überschätzen. Nicht jede »Laboratorisierung « führt zu einer einschneidenden und irreversiblen Transformation eines öffentlichen Problems. Statt eine einzige Zäsur in den Vordergrund zu stellen, statt also den Einfluss der (Labor-)Wissenschaft von vornherein als einmalig und umfassend einzuschätzen, bleibe ich im Folgenden beim historischen Format der Untersuchung und unterziehe Episode für Episode einer laborpragmatisch orientierten Analyse. Eine griffige Formulierung von Bruno Latour abwandelnd, suche ich nicht nur in einem Labor nach anderen

49 »Tod im Vorüberfahren. Im Prozess gegen den Autobahnraser Rolf F. zeigte sich, in welcher Gedankenwelt manche Fahrer leben, die auf deutschen Straßen Autos testen «; Sabine Rückert in DIE ZEIT vom 19. Februar 2004.

50 Hood 2002. 
Mitteln der Politik. Im Anschluss an seine mit Dominique Linhardt vorgetragenen Überlegungen zum Problem der Verkehrssicherheit ${ }^{51}$ und im Unterschied zur symbolpolitischen Deutung suche ich diese Mittel in der Infrapolitik.

\section{Eine infrapolitische Deutung}

Wer die Praxis des Prüfens zum Gegenstand erklärt, kommt nicht um Feldforschung umhin und damit um singuläre Konstruktionen und Arrangements der Kontaktaufnahme, des Dialogs, der Konfrontation und der Aushandlung mit einem konkreten Feld, einem Milieu, einer Szene. Dieser Vorgang wurde oft als temporäre Sozialisation und prekäre Mitgliedschaft reflektiert. In meinem Fall kann davon nur eingeschränkt die Rede sein. Mein Zugriff erfolgt insofern indirekt, als ich ein ausführliches Interview mit einer exponierten Person dieses Feldes zum wichtigsten Fund erkläre. Dieses biografische Narrativ erfüllt, wie ich dargelegt habe, eine zweite methodische Anforderung. Es gibt Aufschluss über Kontinuitäten und Zäsuren in der Praxis des Prüfens. Es bietet Anhaltspunkte für zwei kontrastierende Deutungen, die ich Punkt für Punkt einander gegenüberstelle.

Gegenüber riskanten Techniken, die weitgehend abgeschlossen hinter den Mauern von Großbetrieben eingesetzt werden, muss beim Straßenverkehr eine Besonderheit in Rechnung gestellt werden: Autos werden überwiegend von Laien und auf allgemein zugänglichen Verkehrsflächen betrieben. Das hat Auswirkungen auf die Architektur von Prüfungen - und spricht einmal mehr dafür, die Aufmerksamkeit von Organisationen und organisierten Prüfungen auch auf weniger organisierte und kaum integrierbare Prüfungen auszudehnen. Wie lassen sich Millionen von Laien und von Laien gefahrene Fahrzeuge überwachen und prüfen? Wie flächendeckend und kontinuierlich können diese Prüfungen gewährleistet werden? Diese Frage ist sehr breit und zu offen formuliert, um hier bearbeitet werden zu können. Es soll jedoch festgehalten werden, dass das Alltagsverständnis derer, die am Verkehr »teilnehmen «, durch ein Konglomerat von Prüfungen geprägt ist: Führerscheinprüfung, TÜV, Geschwindigkeitskontrollen, Alkoholtests, Polizeistreifen an Unfallschwerpunkten usw. Es gibt mehr und weniger stationäre, mehr und weniger technisierte, mehr und weniger organisierte Prüfungen; es gibt Prüfungen, die einer externen Instanz bedürfen, und solche, die von den Fahrerinnen und Fahrern (hier und da mit technischer Assistenz) selbst erledigt werden: permanent ein prüfender Blick in den Rückspiegel und nach vorn, ob die Geschwindigkeit an die Fahrbahn, die Witterung und die Verkehrsverhältnisse » angepasst « ist. $^{52}$

Schon bei einer solchen kursorischen Auflistung wird deutlich, dass Aussagen über die Systematik und den Zusammenhang dieser Prüfungen sehr schwer zu treffen sind. Ebensowenig dürfte es möglich sein, ein Maß für die Prüfdichte anzugeben,

51 Latour, Linhardt 2005.

52 In einem weit gestreckten Kontinuum von Prüfungen bedeutet Verkehrssicherheit also auch Normverinnerlichung in großem Maßstab und insofern einen fortgesetzten »Prozess der Zivilisation« (Elias 1976 [1939]). 
ohne sogleich eine Kontroverse anzuzetteln. Für die einen wird zu viel und zu früh, für andere zu wenig und zu spät kontrolliert: $\mathrm{Ob}$ und mit welchen Prüfungen die Sicherheit im Straßenverkehr steigt oder nicht, ist umstritten. Verkehrssicherheit war und ist ganz offensichtlich »ein System ohne Pilot «53, gekennzeichnet durch zahllose, gesunkene und verwachsene Kompromisse zwischen unterschiedlichen Politikfeldern. Auch um besser zu verstehen, wie sich die Rolle staatlicher Politik in diesem fragmentierten Zusammenhang verändert hat, ${ }^{54}$ wende ich mich darum erneut den fünf Episoden zu und rekonstruiere infrapolitische Wendepunkte in der Verkehrssicherheit.

\subsection{Passive Sicherheit erproben}

Die 1966 in den USA in Kraft getretenen Normen für passive Sicherheit stellen eine bedeutende Umstellung dar, die einer infrapolitischen Unterstützung durch Aufpralltest-Laboratorien bedurfte. Herr S. beschreibt dies als einen schrittweise erfolgenden Prozess der "Laboratorisierung « - von Anlagen, die der Witterung ausgesetzt sind, zu überdachten und geschlossenen; von Tierkadavern über Menschenleichen zu standardisierten Testpuppen; vom improvisierten Antrieb zum Testschlitten. ${ }^{55}$ Durch diese Modifikationen wurden auch die Messbedingungen laufend verbessert; zugleich kamen immer präzisere Messinstrumente zum Einsatz, so dass schließlich von gut dokumentierten und replizierbaren Tests die Rede sein konnte. Mit der Stabilisierung von Testanordnungen wird auch die nun als berechenbarer Faktor aufgefasste "Fahrzeugsicherheit « neu konturiert. Die "Fahrgastzelle« wird zu einem epistemischen Objekt; die Frage, wie sich die Kräfte eines Aufpralls über eine Karosserie verteilen und wie sie bei den Fahrzeuginsassen ankommen, wird zu einer Problemstellung, die sich praktisch bearbeiten lässt.

Diese Forschungspraxis bleibt nicht auf die Automobilindustrie beschränkt, sondern wird auch in anderen anwendungsnahen Kontexten betrieben. Über die sektoralen Grenzen hinweg entsteht ein neues Regime der Sichtbarkeit für biomechanische Schnittstellen. Im Nachhinein ist alles an diesem Prozess selbstverständlich, denn was hier entsteht, ist mechanische Objektivität: Partikulare Testobjekte prallen in einer kontrollierten Versuchsanordnung auf universale Testkörper. Herr S. weist darauf hin, dass er über seinen früheren Arbeitgeber und durch mehrere USAReisen schon mit den ersten Testanordnungen vertraut war. Mit der Struktur der für Herrn S. jeweils vordringlichen Aufgaben wanderte auch ihr Ort in der Organisation. Wenn sich Herr S. also kontinuierlich mit Sicherheit beschäftigt hat, dann sind die Gründe dafür nicht in der Organisation (mit stabilen Abteilungen und klar definierten Zuständigkeiten) zu suchen, sondern eher darin, dass er früh ein infrapolitisches Verständnis für Prüfformate und Infrastrukturen des Testens entwickelt und seither gepflegt hat.

53 Galland et al. 2006.

54 Hilse, Schneider 1995; Hamelin et al. 2011.

55 Vgl. Stieniczka 2006, S. 237-241, 254-258. 


\subsection{Diebstahlsicherheit erproben}

Wenn sich Herr S. Jahrzehnte später an die thematisch etwas abseitige Episode der Sicherung gegen Diebstahl erinnert, dann liegt das wohl daran, dass er damals durch ein originelles Testverfahren in Erscheinung getreten ist. Gerade im Vergleich zur vorhergehenden fällt auf, dass diese Prüfung nur bei oberflächlicher Betrachtung laborartige Züge trägt. Sie ist in der personellen Besetzung spektakulär, bleibt aber improvisiert und ist nicht auf Replikation ausgelegt. Die Testanordnung ist mit Kamera und Stoppuhr nur schwach instrumentiert. Es handelt sich also eher um ein Feldexperiment, zumal es keine Hinweise darauf gibt, dass sich diese Testpraxis verstetigt hat. Mit dem Ausgang des Experiments scheint auch das Problem aus der Welt zu sein. Im Unterschied zu allen anderen lässt sich diese Episode auch nicht über die aus dem Forschungsstand geläufigen Periodisierungen absichern und als repräsentativ für die ganze Branche betrachten.

\subsection{Unfallverläufe erproben}

Um 1970 werden in Deutschland jährlich 20.000 Verkehrstote gezählt. Unter dem Eindruck dieser Zahl sah sich die (offizielle) Politik zum Handeln gezwungen. Per Beschluss des Bundestags wurde die Bundesanstalt für Straßenwesen (BASt) zu einem zentralen Institut für Unfallforschung ausgebaut. Mit den Worten einer ehemaligen Leitungsperson sei dieses Instituts dazu da, »öffentlich zu wissen, was bei Unfällen passiert «. Fortan sei mit öffentlichen Mitteln eine »transparente Datenbasis « gelegt worden, die regelmäßig politischen Entscheidungsträgern verfügbar gemacht wird. Mit einem öffentlichen Forschungsauftrag sei sichergestellt worden, dass standardisierte Erhebungsinstrumente zum Einsatz kommen, dass sensible Daten sorgfältig geschützt werden und dass Wissensbestände bereitgestellt werden, die in die Vorbereitung und Evaluation politischer Instrumente und Maßnahmen einfließen - sei es bei verhaltensbezogenen Interventionen (z. B. Gurtpflicht) oder bei der »regelungsnahen « Forschung (z. B. Zulassungsvorschriften).

Das Problem der Verkehrssicherheit erfährt damit einen Schub der »Verwissenschaftlichung ", der zwei aufwendigen Formen epistemischer Praxis besonders zugutekommt: Aufpralltests zur Erforschung »biomechanischer Schnittstellen « und die Verbesserung passiver Sicherheit (vgl. Abschnitt 5.1). Parallel dazu beteiligt sich das Institut am Aufbau einer verbundartigen »Unfallforschung ", für die Herr S. eine prosaische Beschreibung findet: »Was passiert bei einem Unfall? Die Ingenieure gehen zum Auto und rekonstruieren den Unfallhergang. Die Mediziner gehen zu den Verletzten und erhalten Zugang zum Obduktionsbericht. Dann setzen sie sich zusammen und rekonstruieren den Unfallhergang in depth «. Einige Automobilhersteller beteiligten sich an dieser Art der Forschung, allerdings nur für kurze Zeit. Die Ergebnisse solcher Forschungen bleiben für die Fahrzeugentwicklung von untergeordneter Bedeutung. Nach Darstellung von Herrn S. dominiert stattdessen eine Testkultur, die von der Konzernleitung weiterhin als ein unantastbarer Standard betrachtet werde: Nur wer ein hervorragendes Fahrwerk baue, könne es sich erlau- 
ben, schnelle Autos zu verkaufen. ${ }^{56}$ Darum sei nichts wichtiger als der Bremsweg. Wann immer ein neues Modell im Test (und in der populären Fachpresse) in seiner Klasse nicht den kürzesten Bremsweg vorweisen konnte, sei die Entwicklungsabteilung erheblich unter Druck gesetzt worden. Die Persistenz dieses Testkriteriums steht für ein Festhalten an einem Prinzip aktiver Sicherheit: als könnten Unfälle auf diese Weise am besten vermieden werden, obwohl sich, auf politische Initiative und mit finanzieller Unterstützung aus öffentlichen Mitteln, Verfahren und Routinen der Labor- und der Feldforschung etablieren, die das anspruchsvollere, aber symbolpolitisch weniger attraktive Prinzip passiver Sicherheit verlangen. Der Ausbau öffentlicher Forschungsstrukturen hat nicht dazu geführt, dass alle Autohersteller die für sie relevanten Prüfkriterien umstellen.

Dieser Misserfolg lenkt die Aufmerksamkeit noch einmal darauf, dass die Etablierung der genannten Prüfungen, sowohl des Aufpralltests als auch der detaillierten Unfallforschung, ein langwieriger Prozess ist. Zudem sind sie für Probleme anfällig, die für Feldforschung bzw. für Laborforschung typisch sind. Auf der einen Seite fehlt es an Reproduzierbarkeit (Unfallforschung), auf der anderen Seite steht die Repräsentativität in Frage (Crashtests). Hier werden Unfälle als singuläre Ereignisse dokumentiert, dort werden Zusammenstöße nach vorgegebenen Versuchsanordnungen manipuliert. Das hat zur Konsequenz, dass Ergebnisse, die aus einem Format der Prüfung hervorgehen, aus Sicht des jeweils anderen geschwächt werden können. Je stärker also Tests und Prüfungen den genannten, schwer vereinbaren Anforderungen von Reproduzierbarkeit und Repräsentativität unterliegen, ${ }^{57}$ desto leichter können sie gegeneinander ausgespielt werden.

\subsection{Podiumserprobt}

In den 1990er Jahren dehnte sich das Tätigkeitsgebiet von Herrn S. so weit aus, dass er mit fahrzeugbezogener Sicherheitsforschung eigentlich nichts mehr zu tun hatte. Er war zunächst mit dem Aufbau und dann mit der Leitung einer Abteilung mit bis zu 15 Personen befasst, deren Aufgabe darin bestand, alle »Politikthemen « zu bearbeiten, die bisher » nebenher gelaufen « seien.

Der Konzern sei zu der Einsicht gelangt, sich der öffentlich vorgetragenen Kritik am Automobil auch in der Öffentlichkeit stellen zu müssen. Die Öffentlichkeit verlange zu Recht nach technischen Innovationen. Wer allerdings glaube, diese könnten über Nacht in Serie gehen, müsse sich an die langen Produktzyklen in der Autoindustrie erinnern lassen. Es sei einfach zu viel verlangt, antizyklisch zur Produktentwicklung schwierige und teure Nachrüstungen vorzunehmen. Zudem sei es ein Fehler, zu glauben, grundlegende und sehr kostenträchtige Innovationen könnten bei allen Fahrzeugklassen gleichzeitig zum Durchbruch kommen. Die Geschichte habe immer wieder das Gegenteil bewiesen. Gerade neue Sicherheitskomponenten seien

$56 \mathrm{Ob}$ das Bremsweg-Kriterium auch bei anderen Herstellern angewendet wurde, ist mir nicht bekannt. Es lässt sich jedoch unter einen Diskurs der Innovationsverpflichtung subsumieren, der für die deutsche Autobranche überaus bedeutsam ist (Meyer 2009).

57 Vgl. Downer 2007. 
stets zuerst bei Fahrzeugen der Oberklasse eingeführt worden, um dann nach unten »durchzusickern «.

Nur wer diesen Ausführungen folgen kann, wird Herrn S. zustimmen, dass die Autohersteller »ihre Hausaufgaben gemacht " haben. Er wiederholt diesen Satz mehrfach, um dann grundlegende Zweifel an dem Personal in den »Prüfbehörden « zu äußern. Er habe dort mit Leuten zu tun gehabt, die derart fachfremd seien, dass sein Unternehmen sogar Initiativen zu ihrer Weiterbildung ergriffen habe. Personal, das für die Bereiche Wasserwirtschaft oder Bahntechnik qualifiziert sei, könne doch nicht die Zuständigkeit für Autos übertragen werden! Den erhobenen Forderungen nach strengen Auflagen für Umwelt, sicheren Straßen und bewohnbaren Innenstädten habe er die Frage entgegengesetzt: Wer prüft die Prüfer? Mit den exponierten Vertretern der Anti-Auto-Bewegung habe sich sein Unternehmen auf einen wenig zimperlichen Umgang vorbereitet. Um deren Forderungen nach Grenzwerten und Standards zu entkräften, seien Dossiers über diese Personen angelegt worden. Wenn nötig, hätte man ausreichend Material gehabt, um sie mit gezielten Kampagnen rasch zu unglaubwürdigen Zeugen zu machen.

\subsection{Die Sicherheit der anderen erproben}

Nachdem eine längere Phase erhitzter Auseinandersetzungen abgeklungen war, wurden viele Autohersteller von einer Initiative überrascht, die über den Weg der Verbraucherinformation neue Sicherheitsstandards nach sich zog. Die Einsicht, dass auch die Sicherheit anderer Verkehrsteilnehmer - Kinder auf dem Rücksitz und Fußgänger - zu prüfen ist, hat dazu geführt, ein dynamisches Testdesign zu entwickeln.

Allerdings wurden gegen die neuen Testverfahren auch Vorbehalte laut. Das öffentlich geförderte Forschungsprojekt, aus dem EuroNCAP hervorgegangen ist, sei nur eine Fassade, hinter der sich unschwer ein privates Ingenieurbüro mit Sitz in England erkennen lasse. Für die Kritiker ist das Beleg genug, dass dieser Test schon von Anfang an kontaminiert ist, also weder Neutralität noch Wissenschaftlichkeit beanspruchen könne. Es handele sich um einen neuen Versuch, mit der Eigenzeit von Entwicklungszyklen zu brechen. Genau dies sei politisch motiviert, kurzsichtig und gefährlich: Um zu gewährleisten, dass im Rahmen ausgedehnter Entwicklungsprozesse verlässlich geprüft werde, bedürfe es stabiler Bedingungen. ${ }^{58}$

Befürworter des EuroNCAPs, darunter auch die dem Verkehrsministerium zugeordnete Bundesanstalt für Straßenwesen, betonen stattdessen, eine dosierte Öffnung des Labors bewirkt zu haben. Seither sei es möglich, den Test und die Bewertungskriterien nach und nach auf den neuesten Forschungsstand und auf den aktuellen

58 Weiterhin wurde der Test für einen Trend zur »negativen Optimierung « verantwortlich gemacht: Die Hersteller hätten allen Grund, Fahrzeugsicherheit immer nur dort zu erforschen und weiterzuentwickeln, wo es Sternchen und die Aussicht auf eine gute Platzierung gibt. Schließlich muss sich auch die inklusive Testpraxis des EuroNCAP die Kritik gefallen lassen, nur die Sicherheit beim Aufprall auf stehende Hindernisse zu testen. Die Frage der »Kompatibilität« (bei Zusammenstößen unterschiedlicher Fahrzeugtypen) wird dagegen vernachlässigt.

Leviathan, 40. Jg., 4/2012 
Stand der politischen Auseinandersetzung zu heben. Diese Öffnung erfolge jedoch nicht abrupt, sondern nach einem Prinzip des safe landing ${ }^{59}$, so dass die Autohersteller sehr wohl Gelegenheit hätten, sich auf neue und strengere Testkriterien einzustellen. Somit sei außerdem sichergestellt, dass frühere Testergebnisse nicht urplötzlich entwertet würden, was der Glaubwürdigkeit dieses Verfahrens insgesamt abträglich wäre. Darum würden alle Testergebnisse mit einem Jahrgang indiziert; die Anzahl der zu vergebenen Sterne (also die Höchstnote) bleibe jedoch trotz veränderter Testanordnungen konstant. Hier wurde also versucht, eine dynamische Infrastruktur des Testens mit gleichbleibenden symbolpolitischen Aufbauten zu kombinieren.

\section{Fazit: Ein (neues) Zeitalter der Sicherheit?}

Bisher stehen sozialwissenschaftliche Forschungen über Sicherheit im Kontext von großen Thesen zu Transformationen von Staatlichkeit und damit korrespondierenden Prozessen der Subjektivierung. ${ }^{60}$ Sie werden zuweilen und noch zu wenig systematisch ergänzt durch Arbeiten, die sich auch der Risikobearbeitung in und durch Unternehmen widmen. Sofern diese, wie hier gezeigt, den Schwerpunkt auf Prüfungen legen, sind sie noch mehr als die vorher genannten auf eine symbolpolitische Perspektive festgelegt. Nirgendwo zeigt sich das deutlicher und durchgängiger als in den Forschungen von Michael Power über »Rituale der Verifizierung «61, »Das Risikomanagement von Allem [und Jenem] «62, die Erfindung eines »Vorstandsmitglieds für Risiken « ${ }^{63}$, Organisierte Ungewissheit « 64 bis hin zur »Verbetriebswirtschaftlichung der Sicherheit « ${ }^{65}$. Die Frage nach der Infrapolitik technischer Prüfungen stellt sich vor dem Hintergrund dieser düsteren Diagnosen vom Politikverlust gar nicht mehr. Die symbolpolitische Deutung bietet jedoch wenig Raum für Analysen zum Gestaltwandel des Politischen. Darum habe ich einen Scheinwerfer in Gegenrichtung installiert. Während symbolpolitische Deutungen unterstellen, dass sich Praktiken des Prüfens auf zeit- und ortlose Vorgänge der symbolischen Repräsentation reduzieren lassen und dass ihre zeitliche Ausdehnung und ihre Lokalisierung unterschlagen werden können, heben infrapolitische Deutungen auf unterschiedliche Techniken und Formate der zeitlichen, räumlichen und materiellen Verdichtung von konkreten Tests und Prüfungen ab. Eine pragmatische Perspektive auf die Infrapolitik des Prüfens erlaubt, zum Gegenstand der Analyse zu machen, was andernfalls als Symbolpolitik abgetan wird: die zunehmende »Evidenzbasie-

59 Vgl. Pressemitteilung 17/2009 der Bundesanstalt für Straßenwesen. www.bast.de/.../ presse/...pressemitteilung.../2009 (Zugriff vom 28.10.2012).

60 Beck 1986; Ewald 1993.

61 Power 1997.

62 Power 2004.

63 Power 2005.

64 Power 2007.

65 Power 2012. 
rung " der Politik. Es ergibt sich dann eine Version, die ein wechselvolles Hin und Her zwischen unterschiedlichen Formaten der infrapolitischen Verdichtung freilegt. Um dies zu zeigen, ziehe ich nun, von der komplettierten Übersicht ausgehend, einige Quervergleiche.

\begin{tabular}{|c|c|c|}
\hline Karrierestation & Symbolpolitik? & Infrapolitik? \\
\hline 3.1 Marktzugang & $\begin{array}{l}\text { 4.1 Sicherheit und re-inszenierte Tech- } \\
\text { nikgeschichte }\end{array}$ & $\begin{array}{l}\text { 5.1 Passive Sicherheit } \\
\text { erproben }\end{array}$ \\
\hline 3.2 Schließtechnik & $\begin{array}{l}\text { 4.2 Sicherheitstechnische Lösung für } \\
\text { wohl inszenierte Probleme }\end{array}$ & $\begin{array}{l}\text { 5.2 Diebstahlsicherheit } \\
\text { erproben }\end{array}$ \\
\hline 3.3 Unfallforschung & $\begin{array}{l}\text { 4.3 Wohl inszenierte Forschung für } \\
\text { technische Lösungen }\end{array}$ & $\begin{array}{l}5.3 \text { Unfallverläufe erpro- } \\
\text { ben }\end{array}$ \\
\hline $\begin{array}{l}3.4 \text { Öffentlichkeits- } \\
\text { arbeit }\end{array}$ & 4.4 Re-Delegierung & 5.4 Podiumserprobt \\
\hline $\begin{array}{l}3.5 \text { Verbraucher- } \\
\text { information }\end{array}$ & $\begin{array}{l}4.5 \text { Die Inszenierung mechanischer } \\
\text { Objektivität }\end{array}$ & $\begin{array}{l}5.5 \text { Die Sicherheit der } \\
\text { anderen erproben }\end{array}$ \\
\hline
\end{tabular}

In Europa wurden Risiken des Straßenverkehrs erst über den Umweg der USGesetzgebung zu einem Problem der Fahrzeugsicherheit. Mit der Ankunft dieses Problems bei der Autoindustrie beginnt die hier aufgearbeitete Geschichte. Die "Ankunft « ist aber keine einmalige Sache; das Problem kommt immer wieder, in wechselnder Gestalt, und es wird auf unterschiedliche Weise verarbeitet. In der Folge überlagern sich Problemdefinitionen und existieren mit wechselnden Schwerpunktsetzungen nebeneinander her. Wenn die Industriegesellschaft nicht als eine Gesellschaft großer Organisationen, sondern als eine Gesellschaft technischer Prüfungen betrachtet wird, dann fällt auf, dass sich die Orte der laborförmigen Prüfungen für Fahrzeugsicherheit vermehrt haben. Geprüft wird als Bedingung für den Marktzugang (3.1) und zur (nachträglichen) Verbraucherinformation (3.5). Wie ist das zu interpretieren, wenn nicht als eine Bewegung von technischen zu weniger technischen, von wissenschaftlich abgesicherten zu weniger fundierten Prüfungen? Kann ein Labor, das für Prüfungen vor der Zulassung zuständig ist, überhaupt mit einem Labor verglichen werden, das der Zulassung nachgelagert ist und nur noch der Verbraucherinformation dient?

Wer so argumentiert, nähert sich wieder der symbolpolitischen Lesart und wird der Phase der Öffentlichkeitsarbeit (3.4) eine Schlüsselrolle zuerkennen. Die oben rekonstruierte Argumentationslinie der Konzerne könnte sich demnach als wirksam herausgestellt haben: Erstens gefährdet, wer in den Produktzyklus eingreift, die Innovation. Zweitens sind alle - auch die Sicherheit betreffenden - Prüfungen Teil eines komplexen und fragilen Entwicklungszyklus, der gegen regulierende Eingriffe zu schützen ist. Drittens sind die existierenden Prüfungen im Rahmen der Zulassung starr und darum innovationsfeindlich. Viertens kommt zum bürokratischen Dickicht dieser Prüfungen noch die fehlende Sachkenntnis des prüfenden Personals. Fünftens sind alle Kritiker, Expertinnen und Aktivisten, die neuartige Prüfungen 
anstoßen könnten, gegebenenfalls anzuschwärzen. Fast alle Versatzstücke dieser Argumentationslinie sind aus anderen Diskussionszusammenhängen gut bekannt, angefangen bei der Bürokratiekritik bis hin zu Kontroversen und Auffassungen zur Entstaatlichung und Privatisierung der Sicherheit. ${ }^{66}$ Ist der Hinweis auf Infrapolitik und unterschiedliche Konfigurationen der Laboratorisierung darum allenfalls ein Umweg?

Wer Prüfungen eine besondere Stellung einräumt und sich in die Lage versetzt, diese präzise zu unterscheiden und begrifflich zu fassen, muss auch die vierte Episode einer stärker infrapolitischen Deutung unterziehen. Der Akzent läge dann weniger darauf, dass bestimmte Akteure (hier: die Autokonzerne) mit neuartigen Mitteln ihre bekannten Interessen verfolgen, sondern darauf, dass und wie Mittel und Interessen aus einer Praxis des Prüfens hervorgehen. Zum anderen wäre der Unfallforschung (3.3) besonderes Gewicht zu geben. Den an Laborforschung angelehnten Aufpralltestverfahren erwächst durch die Unfallforschung Konkurrenz; diese Konkurrenzsituation lässt eine Bruchstelle zwischen Testverfahren aufscheinen, die sich beide auf wissenschaftliche Standards berufen. Vor diesem Hintergrund wird eine doppelte Leistung des EuroNCAP (3.5) deutlich. Er hat nicht nur als ein Instrument der Inklusion gewirkt, indem er ein geschlossenes Testverfahren aufgebrochen und darüber die Öffentlichkeit wieder ins Labor geholt hat. Er hat auch einen Kompromiss zwischen zwei inkompatiblen Verfahren des Testens möglich gemacht, der sich als ziemlich haltbar erweist. Insofern kann keine Rede davon sein, dass dieser Test »nur« den Ort der Prüfung von vorn (Zulassungsvorschriften) nach hinten (Verbraucherinformation) verschoben hat.

Die infrapolitische Deutung beobachtet jeweils kontingente Sicherheitserfordernisse aus dem Blickwinkel der Entstehung konkreter und praktischer Verfahren und Routinen des Prüfens und Testens. Diesen Praktiken wird eine eigene Temporalität zugestanden. Sie können schwer und träge sein und darum neue Problemdefinitionen verzögern. Aus der Perspektive der Infrapolitik hat die letzte Station - die Einführung eines dynamischen Tests (EuroNCAP) - auch darum einen herausgehobenen Status. Dieses Testverfahren ist nicht träge; es ist vielmehr so programmiert, dass es mit der Zeit immer strengere Kriterien anlegt.

Die symbolpolitische Deutung hebt dagegen eine andere Kontinuität hervor. Gewiss, es stellt sich in jeder Episode eine neue Herausforderung. Aber es sind nicht neuartige Testverfahren, die sie zum Abschluss bringen. Vielmehr wird Fall um Fall gezeigt, wie diese Prüfungen symbolpolitisch degradiert und auf diese Weise für ein durchgängig wirksames Kriterium anschlussfähig werden, das nicht lange gesucht werden muss: Geprüft und getestet wird nur so, dass die Kasse stimmt. Ob lokal und improvisiert oder translokal und stark institutionalisiert, die Integration aller möglichen Testverfahren in ein ökonomisches Kalkül erscheint aus dieser Sicht möglich und wahrscheinlich. Prüfungen, die für (mehr) Sicherheit sorgen sollen, finden dann kaum Beachtung. Gleich wie sie aussehen, die Anstrengungen, die Realität einer bedrohlichen Lage überprüfbar zu machen, verdienen nur so lange Aufmerksamkeit, bis ein symbolpolitischer Vorbehalt geltend gemacht werden kann. 
Diese Vorgehensweise ist von vornherein ideologiekritisch; es steht immer schon fest, dass Prüfungen nur inszeniert sind. Sie tragen zum Verfall des Politischen bei; selbst die raffinierteste Testanordnung ist nicht in der Lage, diesen aufzuhalten.

Dem ist aus infrapolitischer Perspektive zu entgegnen, dass Prüfungen zum Gestaltwandel des Politischen beitragen. Nicht etwa, weil sie im Namen der Sicherheit abschließend objektive Ergebnisse hervorbringen. In der infrapolitischen Deutung lässt sich der Vorzug einer pragmatischen Sichtweise ausspielen. Statt sich vorab auf Naivität (eine Prüfung bringt ein abschließendes Ergebnis) oder auf Verdacht (eine Prüfung ist nur inszeniert) festzulegen, bemüht sie sich, Ökonomien des Prüfens zu erkunden. Sie verlagert das Problem dazu zunächst auf die Ebene der Akteure und deren Mittel und Wege, Prüfungen einzufordern, auszulösen, aufrechtzuerhalten, zu wiederholen, zu kritisieren, aber auch abzuschließen. Der letzte Aspekt ist zu unterstreichen: Ohne Ökonomien des Prüfens wäre der Verdacht ein (unerträglicher) Dauerzustand. Aus pragmatischer Perspektive werden Prüfungen als situierte Prozesse begriffen und zum Gegenstand konkreter Beschreibungen gemacht. Wenn dabei diverse Messgeräte, Prüfinstrumente und Experimentalverfahren zum Einsatz kommen, scheint mir die Wortschöpfung »Laborpragmatismus « treffend. Aus infrapolitischer Perspektive ist also auf die Inkommensurabilität mehrerer Ökonomien des Testens und Prüfens zu insistieren. In der Gegenüberstellung wurde deutlich, dass sie Orte, Formate und Praktiken des Prüfens benennt, die in der symbolpolitischen Lesart unterschlagen werden.

Wenn die Industriegesellschaft vornehmlich eine Gesellschaft von Organisationen ist, dann hat sich über die Zeit der hier betrachteten Problematisierungen der Verkehrssicherheit wenig geändert. Die Automobilunternehmen sind eher größer und mächtiger geworden. Wer Industriegesellschaften stattdessen auch über einen bestimmten Typ von Prüfungen identifiziert, dem bietet sich ein Blick auf eine markante Veränderung: Mit den Prüfungen haben sich die Bedingungen, unter denen sich Organisationen als überlebensfähig beweisen müssen, stark gewandelt, und zwar, wenn man von der Autoindustrie ausgeht, zugunsten großer Organisationen, die in der Lage sind, sich rasch auf neue Prüfungen einzustellen.

Die Automobilindustrie war für mindestens eine Generation von Soziologinnen und Soziologen ein emblematischer Gegenstand, der Theorieentwicklungen ermöglicht und greifbar gemacht hat. In der Folge lagen Forschungen zum Verhältnis von Autoindustrie und Gesellschaft eine Zeit lang brach. Wenn sich diese Kontinuität wiederentdecken, neu erschließen und pflegen lässt, dann über eine politische Soziologie technischer Prüfungen. Entgegen einer symbolpolitischen Lesart, die ihn schon als abgeschlossen betrachtet hat, liefert der hier betrachtete Fall der automobilen Sicherheit ein infrapolitisches Lehrstück.

\section{Literatur}

Adams, John 1985. Risk and freedom. The record of road safety regulation. Nottingham: Bottesford Press.

Bardet, Fabrice 2008. "Quand les constructeurs automobiles façonnent la sécurité routière. La médiatisation des accidents de la route aux Etats-Unis «, in Réseaux 147, S. 87-113.

Beck, Ulrich 1986. Risikogesellschaft. Frankfurt a. M.: Suhrkamp.

Leviathan, 40. Jg., 4/2012 
Beck, Ulrich 1993. Die Erfindung des Politischen. Frankfurt a. M.: Suhrkamp.

Bell, Daniel 1973. The coming of post-industrial society. London: Heinemann.

Birkland, Thomas A. 2004. »Learning and policy improvement after disaster. The case of aviation security ", in American Behavioral Scientist 48, 3, S. 341-364.

Boltanski, Luc; Chiapello, Eve 2003. Der neue Geist des Kapitalismus. Konstanz: UVK.

Boltanski, Luc; Thévenot, Laurent 2007. Über die Rechtfertigung. Eine Soziologie der kritischen Urteilskraft. Hamburg: Hamburger Edition.

Boltanski, Luc 1990. "Sociologie critique et sociologie de la critique", in Politix 10-11, S. 124-134.

Breviglieri, Marc; Lafaye, Claudette; Trom, Danny 2009. »Sociologie pragmatique et normativité de l'agir en public ", in Compétences critiques et sens de la justice, hrsg. v. Breviglieri, Marc; Lafaye, Claudette; Trom, Danny, S. 7-12. Paris: Economica.

Cefaï, Daniel 2009. "La fabrique des problèmes publics. Boire ou conduire, il faut choisir «. Nachwort zu La culture des problèmes publics. L'alcool au volant: la production d'un ordre symbolique (Joseph Gusfield), S. 219-318. Paris: Economica.

Downer, John 2007. "When the chick hits the fan: representativeness and reproducibility in technological testing ", in Social Studies of Science 31, 1, S. 7-26.

Edelman, Murray J. 1964. The symbolic uses of politics. Urbana: Illinois University Press.

Elias, Norbert 1976 [1939]. Über den Prozess der Zivilisation. Frankfurt a. M.: Suhrkamp.

Ewald, François 1993. Der Vorsorgestaat. Frankfurt a. M.: Suhrkamp.

Florida, Richard L. 2002. The rise of the creative class: and how it's transforming work, leisure, community and everyday life. New York: Basic Books.

Galland, Jean-Pierre et al. 2006. "La sécurité routière: une vision restrictive, un système sans pilote? ", in Cahiers risques collectifs et situations de crise no.6: recherche et sécurité routière. Pour une action publique renouvelée. Publications de la MSH-Alpes, S. 15-30.

Gilbert, Claude 1992. Le pouvoir en situation extrême. Catastrophes et politique. Paris: Harmattan.

Gilbert, Claude 2008. »Quand l'acte de conduite se résume à bien se conduire «, in Réseaux 147, S. 21-48.

Glaeßner, Gert-Joachim 2001. »Sicherheit und Ordnung. Politisch-soziologische Reflexionen zu einem (wieder) aktuellen Thema ", in Berliner Journal für Soziologie 11, 3, S. 337-358.

Guggenheim, Michael 2012. "Laboratizing and delaboratizing the world: changing sociological concepts for places of knowledge production ", in History of the Human Sciences 25, 1, S. 99-118.

Guggenheim, Michael; Potthast, Jörg 2012. »Symmetrical twins. On the relationship between ANT and the sociology of critical capacities ", in European Journal of Social Theory 15, 2, S. 157-178.

Gusfield, Joseph R. 1981. The culture of public problems: drinking-driving and the symbolic order. Chicago: Chicago University Press.

Hamelin, Fabrice et al. 2011. Sciences et politiques de la sécurité routière. Comparaisons européennes. Paris: IFSTTAR.

Hasse, Raimund; Krücken, Georg; Weingart, Peter 1994. »Laborkonstruktivismus. Eine wissenschaftssoziologische Reflexion", in Konstruktivismus und Sozialtheorie, hrsg. v. Rusch, Gebhard; Schmidt, Siegfried J., S. 220-261. Frankfurt a. M.: Suhrkamp.

Heintz, Bettina 1998. „Die soziale Welt der Wissenschaft. Entwicklungen, Ansätze und Ergebnisse der Wissenschaftsforschung ", in Wissenschafts- und Technikforschung in der Schweiz, hrsg. v. Heintz, Bettina; Nievergelt, Bernhard, S. 55-94. Zürich: Seismo.

Hilse, Hans-Günter; Schneider, Walter. Hrsg. 1995. Verkehrssicherheit. Handbuch zur Entwicklung von Konzepten. Stuttgart: Richard Boorberg Verlag.

Hood, Chris 2002. "The risk game and the blame game", in Government and Opposition 37, 1, S. 15-37.

Knorr Cetina, Karin D. 1988. »Das naturwissenschaftliche Labor als Ort der >Verdichtung von Gesellschaft ", in Zeitschrift für Soziologie 17, 2, S. 85-101.

Kohler, Robert E. 2002. Landscapes and labscapes. Exploring the lab-field border in biology. Chicago: Chicago University Press.

Koppetsch, Cornelia 2011. »Symbolanalytiker - ein neuer Expertentypus? Einige Thesen zum Wandel akademischer Berufsfelder «, in Leviathan 39, 3, S. 407-433. 
Krohn, Wolfgang; Weyer, Johannes 1990. »Die Gesellschaft als Labor. Risikotransformation und Risikokonstitution durch moderne Forschung ", in Riskante Entscheidungen und Katastrophenpotentiale, hrsg. v. Halfmann, Jost; Japp, Klaus-Peter, S. 89-122. Opladen: Westdeutscher Verlag.

Latour, Bruno 1984. The Les microbes. Guerre et paix suivi de irréductions. Paris: Métalié.

Latour, Bruno; Linhardt, Dominique 2005. L'objet de la sécurité routière. Produire de nouvelles connaissances: de l'assemblage à l'assemblée. Paris: Centre de Sociologie de l'Innovation.

Latour, Bruno; Woolgar, Steve 1986. Laboratory life: the construction of scientific facts. Princeton: Princeton University Press.

Leonardi, Paul M. 2010. »From road to lab to math: the co-evolution of technological, regulatory, and organizational innovations for automotive crash testing ", in Social Studies of Science 40, 2, S. 243-274.

Linhardt, Dominique; Muniesa, Fabian 2011. »Tenir lieu de politique: le paradoxe des spolitiques d'économisation «, in Politix 95, S. 7-21.

Lippmann, Walter 1925. The phantom public. New York: Harcourt Brace.

Meyer, Ulrich, 2009. Innovationspfade. Evolution und Institutionalisierung komplexer Technologie in organisationalen Feldern. Dissertation an der TU Berlin.

Pinch, Trevor 1993. " Testing - one, two, tree ... testing! Toward a sociology of testing «, in Science, Technology \& Human Values 18, 1, S. 25-41.

Potthast, Jörg 2011. »Sense and security. A comparative view on recent changes of access control at airports ", in Science, Technology \& Innovation Studies 7, 1, S. 87-106.

Potthast, Jörg 2012. »Fahrgastzellen ", in Container/Containment. TUMULT, Schriften zur Verkehrswissenschaft, Band 38, hrsg. v. Klose, Alexander; Potthast, Jörg, S. 63-75. Wetzlar: Büchse der Pandora.

Power, Michael 1997. The audit society: rituals of verification. Oxford: Oxford University Press.

Power, Michael 2004. The risk management of everything. London: Demos.

Power, Michael 2005. "Organizational responses to risk: the rise of the chief risk officer ", in Organizational encounters with risk, hrsg. v. Hutter, Bridget; Power, Michael, S. 132-148. Cambridge: Cambridge University Press.

Power, Michael 2007. Organized uncertainty: designing a world of risk management. Oxford: Oxford University Press.

Power, Michael 2012. "The managerialization of security «, in Securitization, accountability and risk management: transforming the public domain, hrsg. v. Svedburg Helgesson, Karin; Mörth, Ulrika, S. 70-87. London: Routledge.

Rabinow, Paul 1996. Making PCR: a story of biotechnology. Chicago: Chicago University Press.

Reich, Robert B. 1992. The work of nations: preparing ourselves for 21st century capitalism. New York: Vintage Books.

Sarcinelli, Ulrich 2011. Politische Kommunikation in Deutschland. Medien und Politikvermittlung im demokratischen System. 3., überarbeitete und erweiterte Auflage. Wiesbaden: VS Verlag für Sozialwissenschaften.

Stieniczka, Norbert 2006. Das »narrensichere « Auto. Die Entwicklung passiver Sicherheitstechnik in der Bundesrepublik Deutschland. Darmstadt: Wissenschaftliche Buchgesellschaft.

Vries, Gerard de 2007. »What is political in sub-politics? How Aristotle might help STS «, in Social Studies of Science 37, 5, S. 781-809.

Weingart, Peter; Krohn, Wolfgang 1986. »>Tschernobyk - das größte anzunehmende Experiment", in Kursbuch 85, S. 1-25.

Wetmore, Jameson M. 2004. »Redefining risks and redistributing responsibilities: building networks to increase automobile safety", in Science, Technology \& Human Values 29, 3, S. 377-405.

Zelinka, Fritz F. 1974. "Zum Selbstverständnis der Soziologie in der Verkehrssicherheitsforschung ", in Kölner Zeitschrift für Soziologie und Sozialpsychologie 26, 4, S. 742-760.

Leviathan, 40. Jg., 4/2012 
Zusammenfassung: Wenn es um Sicherheit geht, sieht die sozialwissenschaftliche Forschung vor allem symbolpolitische Inszenierungen. Auch der Fall der Verkehrssicherheit liefert dafür reichlich Anhaltspunkte. Symbolpolitische Deutungen unterschätzen jedoch den Gestaltwandel des Politischen. Infrapolitische Deutungen heben dagegen auf unterschiedliche Formate von Tests und Prüfungen ab. Der Beitrag erörtert das Spannungsfeld beider Deutungen anhand der Karriereerzählung eines Ingenieurs, der über 30 Jahre lang bei einem Autohersteller mit Sicherheitsfragen befasst war.

Stichworte: Verkehrssicherheit, Automobilindustrie, Prüfung, Infrapolitik, Symbolpolitik

\section{Political sociology of technical testing. The case of road safety}

Summary: In dealing with safety and security issues, social science research has a focus on symbolic policy. The case of road safety offers telling examples. However, interpretations highlighting symbolic uses politics often miss the point of changing policy regimes. To compensate for this shortcoming, the contribution turns to infrapolitics as exerted by various forms of trials and testing. Trying to understand how symbolic policy relates to infrapolitics, it closely analyses the biographical narrative of a former engineer employed by a large car manufacturer and in charge of safety issues for more than 30 years.

Keywords: road safety, car industry, testing, infrapolitics, symbolic policy

Autor

Jörg Potthast

TU Berlin

Institut für Soziologie

Sekr. FH 9-1

Fraunhoferstr. 33-36

10587 Berlin

joerg.potthast@tu-berlin.de 